\title{
Computational Geometry for Sculpture
}

\author{
George W. Hart \\ Sculptor \\ http://www.georgehart.com/ \\ george@georgehart.com
}

\begin{abstract}
This presentation illustrates examples of my geometric sculpture and outlines certain design techniques. I apply methods from the field of computational geometry to the creation of forms which are aesthetic objects.

I am a full-time sculptor creating works that manifest what I call the geometric aesthetic. This aesthetic celebrates the beauty of geometry and spatial rationality. I seek to produce novel forms which engage the viewer visually and have an underlying coherence based on symmetric three-dimensional mathematical structures. Internal relationships between the components of a sculpture can provide a depth to the work, leading the viewer to return again and again, each time seeing deeper into the piece. In addition, the viewer is led to ask questions of a mathematical nature about the patterns he or she finds.

In the design of such sculpture, there are many issues of calculation to be addressed. I work constructively, assembling parts that are either common household objects or carefully formed abstract shapes. Exact lengths and angles must be worked out for these components to fit together appropriately. But there are also many free parameters that I may vary in seeking an ideal design.
\end{abstract}

Physical models are sometimes sufficient, but often I write software to allow me to explore the design space of a sculpture. This makes me a user of computational geometry methods, applying appropriate techniques for each problem. However, unlike most computational geometers, my concerns are for an expedient result which I find aesthetically valid, rather than efficient general purpose algorithms. Iterative numerical methods are often satisfactory.

Figures 1-11 illustrate a variety of recent works and references [1][13] provide some further details.

\section{Categories and Subject Descriptors}

J.5 [Arts and Humanities]: Fine arts. I.3.5 [Computational Geometry and Object Modeling]: Geometric algorithms.

Permission to make digital or hard copies of all or part of this work for personal or classroom use is granted without fee provided that copies are not made or distributed for profit or commercial advantage and that copies bear this notice and the full citation on the first page. To copy otherwise, or republish, to post on servers or to redistribute to lists, requires prior specific permission and/or a fee.

$S C G^{\prime} 01$, June 3-5, 2001, Medford, Massachusetts, USA

Copyright 2001 ACM 1-58113-357-X/01/0006_..\$5.00.

\section{General Terms}

Algorithms.

\section{Keywords}

Art, Sculpture, Computational Geometry, Polyhedron, Polyhedra.

\section{REFERENCES}

[1] George W. Hart, web pages, http://www.georgehart.com/

[2] George W. Hart and Henri Picciotto, Zome Geometry, Key Curriculum Press, 2001.

[3] Craig Kaplan and George W. Hart, "Symmetrohedra" to appear in Proceedings of Bridges 2001: Mathematical Connections in Art, Music and Science, Southwestern College, Kansas, July 2001.

[4] Douglas Zongker and George W. Hart, "Blending Polyhedra with Overlays" to appear in Proceedings of Bridges 2001: Mathematical Connections in Art, Music and Science, Southwestern College, Kansas, July 2001.

[5] George W. Hart, "Loopy," Humanistic Mathematics, to appear, 2001.

[6] George W. Hart, "Reticulated Geodesic Constructions," Computers and Graphics 24(6), 2000.

[7] George W. Hart, "Solid-Segment Sculptures," Proceedings of Colloquium on Math and Arts, Maubeuge, France, Sept. 2000. (to appear)

[8] George W. Hart, "Sculpture based on Propellorized Polyhedra," in Proceedings of MOSAIC 2000, University of Washington, Seattle, August 2000.

[9] George W. Hart, "Millennium Bookball," Proceedings of Bridges 2000: Mathematical Connections in Art, Music and Science, Southwestern College, Kansas, July 2000, and Visual Mathematics 2(3).

[10] George W. Hart, "Zonohedrification," The Mathematica Journal, vol. 7 no.3, 1999.

[11] George W. Hart, "Icosahedral Constructions," Proceedings of Bridges: Mathematical Connections in Art, Music and Science, Southwestern College, Kansas, July 1998.

[12] George W. Hart, "Zonish Polyhedra," Proc. of Mathematics and Design '98, San Sebastian, Spain, June 1998.

[13] George W. Hart, "Calculating Canonical Polyhedra," Mathematica in Research and Education, vol. 6-3, 1997. 


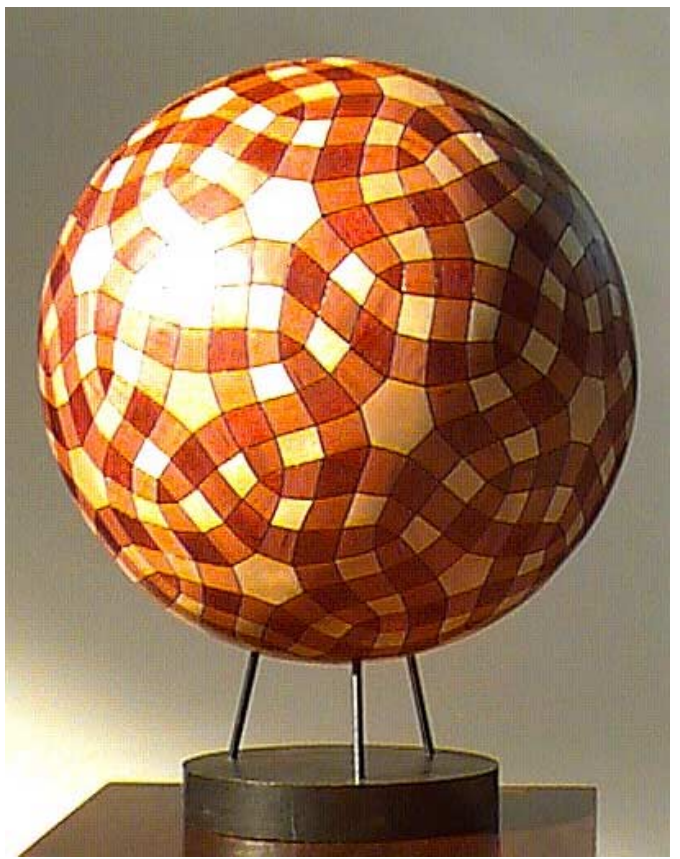

Figure 1. Roads Untaken, wood, 17 inches. [8, 13] The form is the "exploded propellorized truncated icosahedron." A set of polyhedral operators produced the topological structure and a numerical relaxation method was used to proportion it so all edges are tangent to a common sphere. Is there a light path from any hexagon to any other hexagon? Is there a dark path from any triangle to any other triangle?

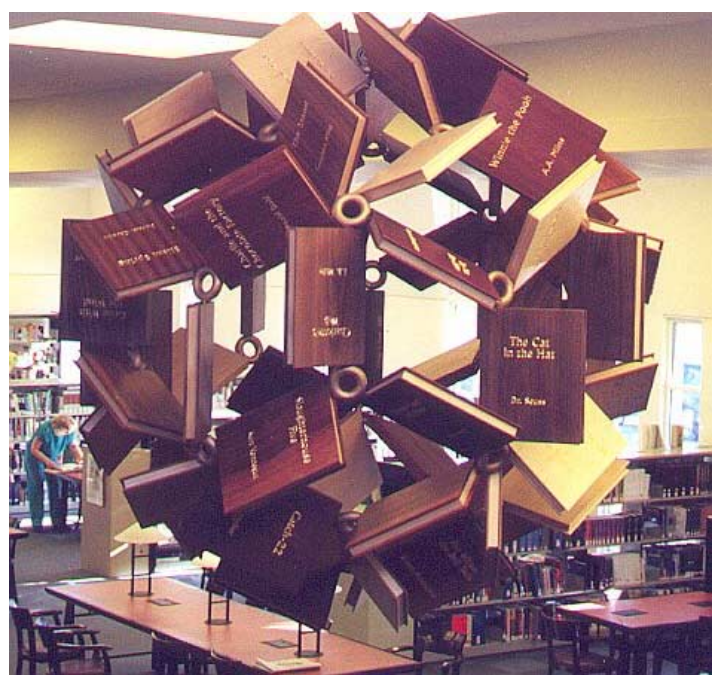

Figure 3. Millenium Bookball, wood and bronze, 5 feet. [9] Commissioned by the Northport Public Library, this sculpture was assembled at a community "barn raising". There are 32 bronze tori connecting the books. The books which meet at any torus form a type of "propeller" There are twenty 3-way propellers and twelve 5-way propellers. The spines of the books follow the edges of a rhombic triacontahedron and the tilt of their planes was chosen so each book is coplanar with another book partway around the other side.

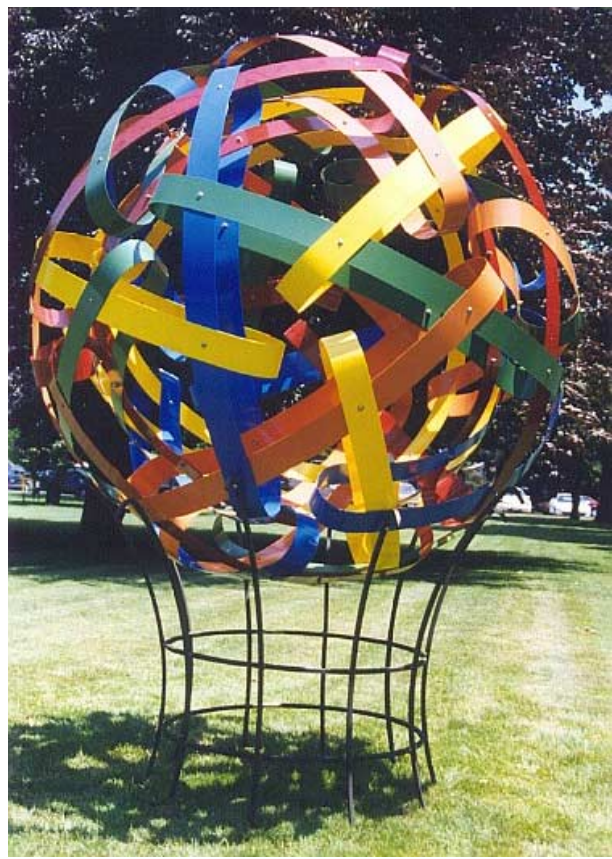

Figure 2. Loopy, painted aluminum, 8 feet. [5] These 30 loops, each 10 feel long if unrolled, are woven through each other and bolted together. The five colors are carefully arranged so that each loop crosses one loop of the other four colors, each color appears once around each 5-sided opening, and the ends of each loop approach the ends of two other loops of the same color.

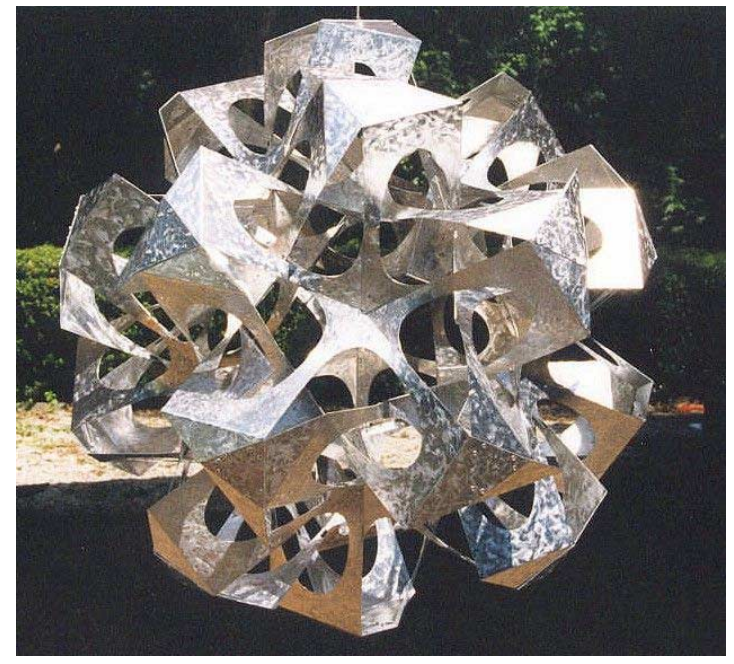

Figure 4. Whoville, aluminum, 35 inches. [11] The form derives from an icosahedron and dodecahedron in mutually dual position, which would lie in the empty central region of the sculpture. The five-fold dimples correspond to the vertices of the icosahedron and the three-fold dimples (in the "basements" of the three-sided buildings) correspond to the vertices of the dodecahedron. The lines of the sculpture extend or parallel the edges of these polyhedra. The rectangular form of each doorway is a golden rectangle and the triangles were chosen to create coplanar pairs. 


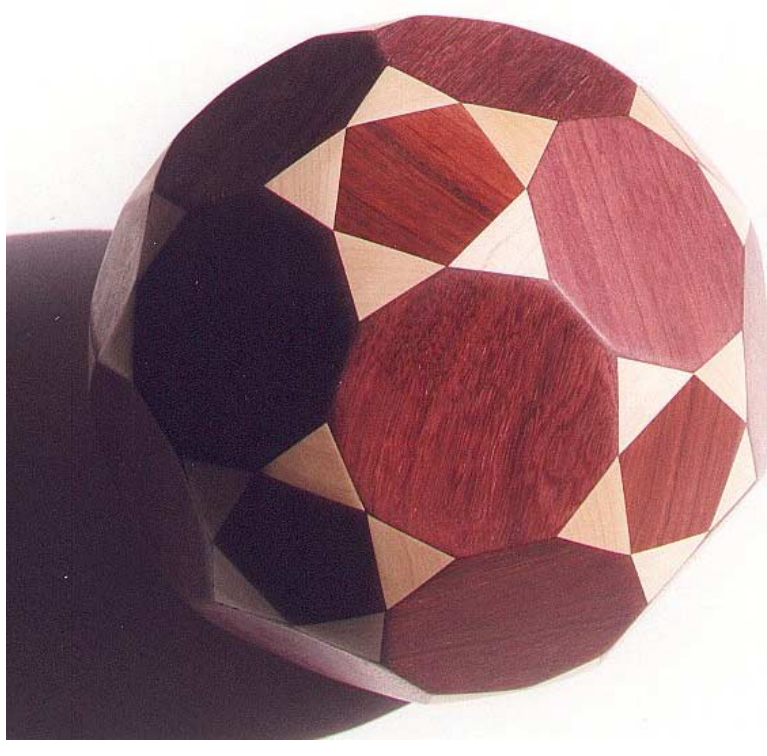

Figure 5. untitled, wood, 12 inches. [3] Produced by an algorithm developed in joint research with Craig Kaplan, this form is unusual in that it incorporates 20 regular enneagons (along with 12 regular pentagons and 60 isosceles triangles). The faces are positioned in space with icosahedral symmetry. This is one of many novel forms we call "symmetrohedra".

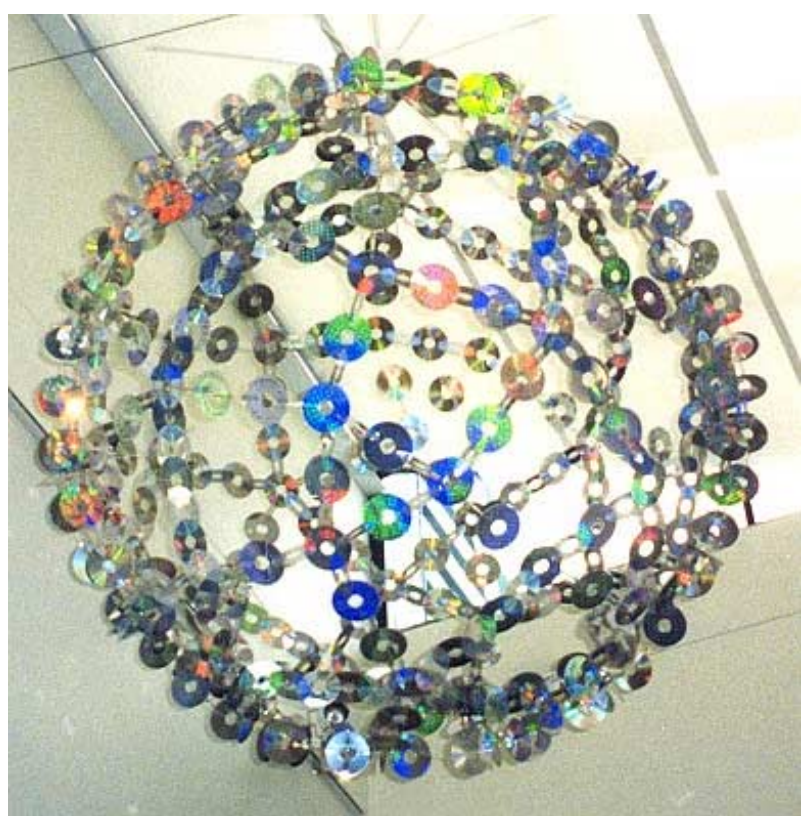

Figure 7. Rainbow Bits, CD ROMs, 6 feet. [8] Carlo Sequin commissioned this orb of 642 holographic CDs for the Computer Science building of U.C. Berkeley. The form is based on the "propello-icosahedron," which consists of 20 equilateral triangles and 60 tri-equiangular "kites." These kites are tetragons with two pairs of equal sides and three equal angles. It was assembled in place, 20 feet up in the Soda Hall atrium where it hangs.

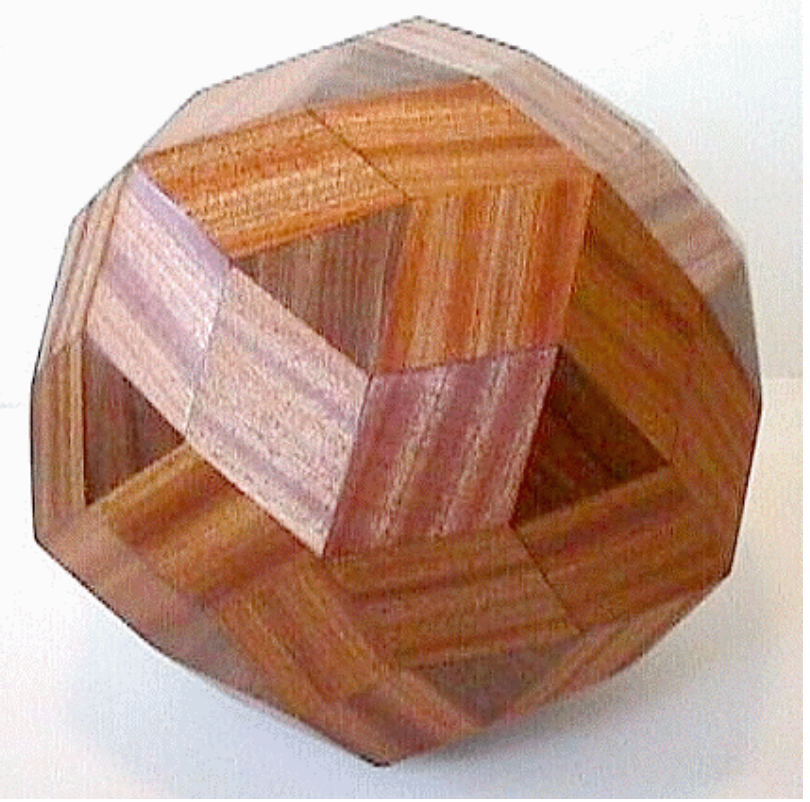

Figure 6. untitled, wood, 12 inches. [4] Produced by an algorithm developed in joint research with Douglas Zongker, this form is surprising because it incorporates 60 identical rhombi and 20 equilateral triangles. It is the Minkowski sum of the five regular tetrahedra that can be simultaneously inscribed in a regular dodecahedron.

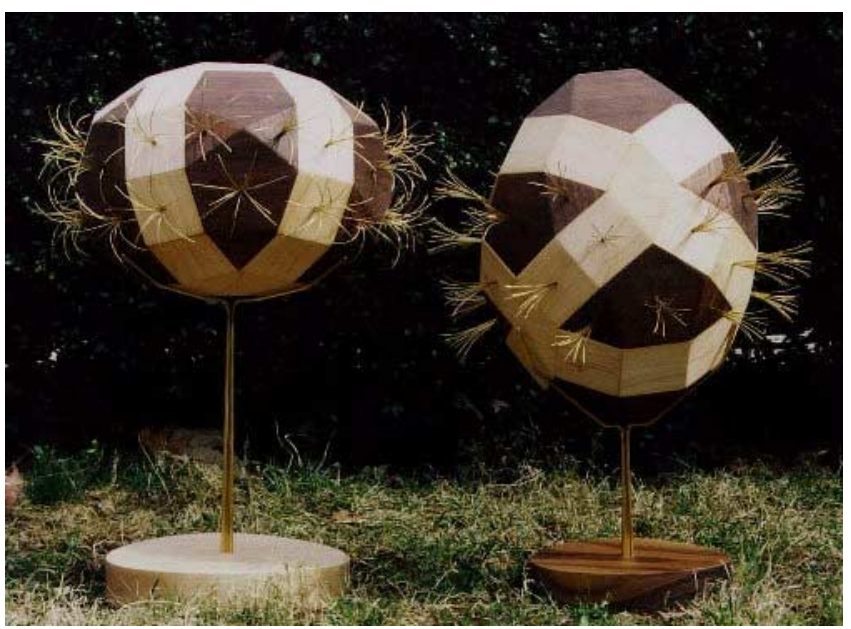

Figure 8. Fat and Skinny, wood and brass, 23 inches. [12] Both are constructed from the same 12 regular pentagons, 30 squares, 20 equilateral triangles, and 6 golden rhombi, but they are arranged differently. In each case, the dark components (pentagons and triangles) fit together as an icosidodecahedron. The light components (squares and rhombi) are arranged along three of the icosidodecahedron's "equators" but there are two ways to choose three equators. 


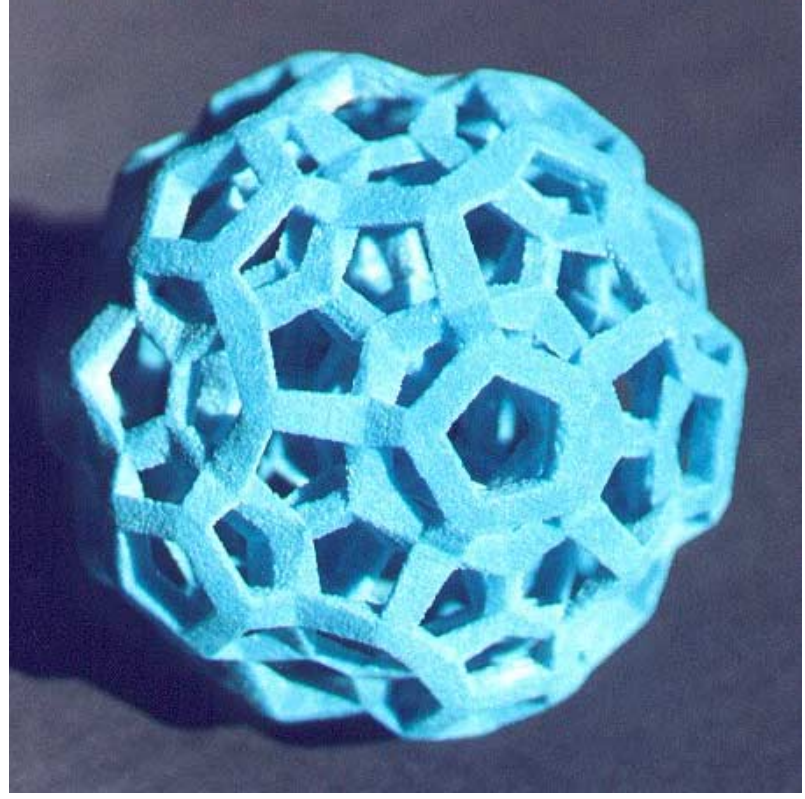

Figure 9. Five-Legged-Bee Hive, 3 inches, 3D printing. [7] This is based on a projection of the regular 4-dimensional polytope called the "120-cell," but adapted into a more textured, chiseled form. Angular, faceted struts were designed to give this work a sculptural character. There are many 5-sided openings and passages throughout its volume. It is made of plaster by an automated 3D printing process, without any human hand. After I design such a sculpture as a computer file, it is fabricated in a machine which scinters, laminates, or solidfies thousands of very thin layers.

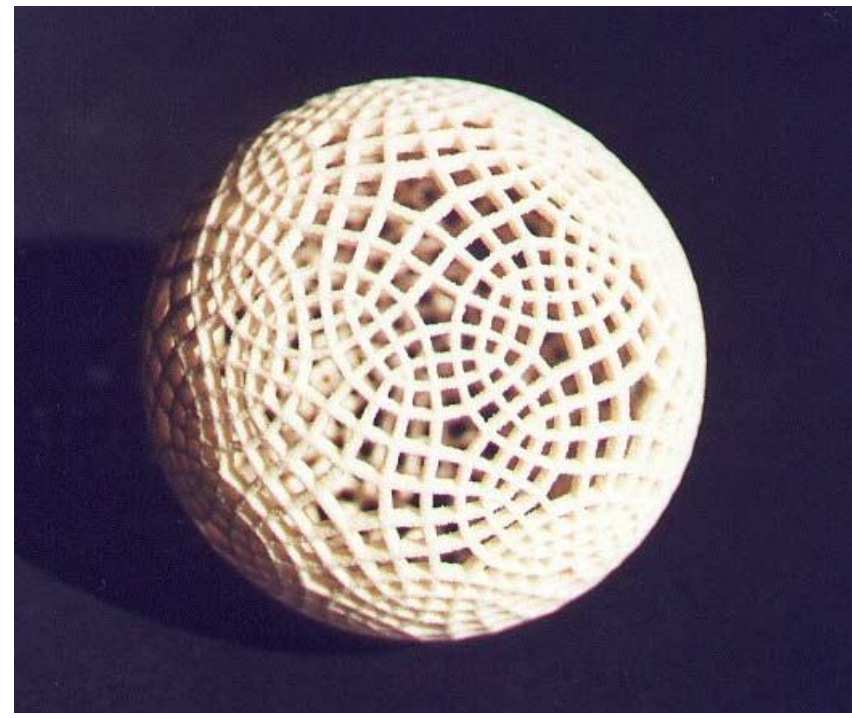

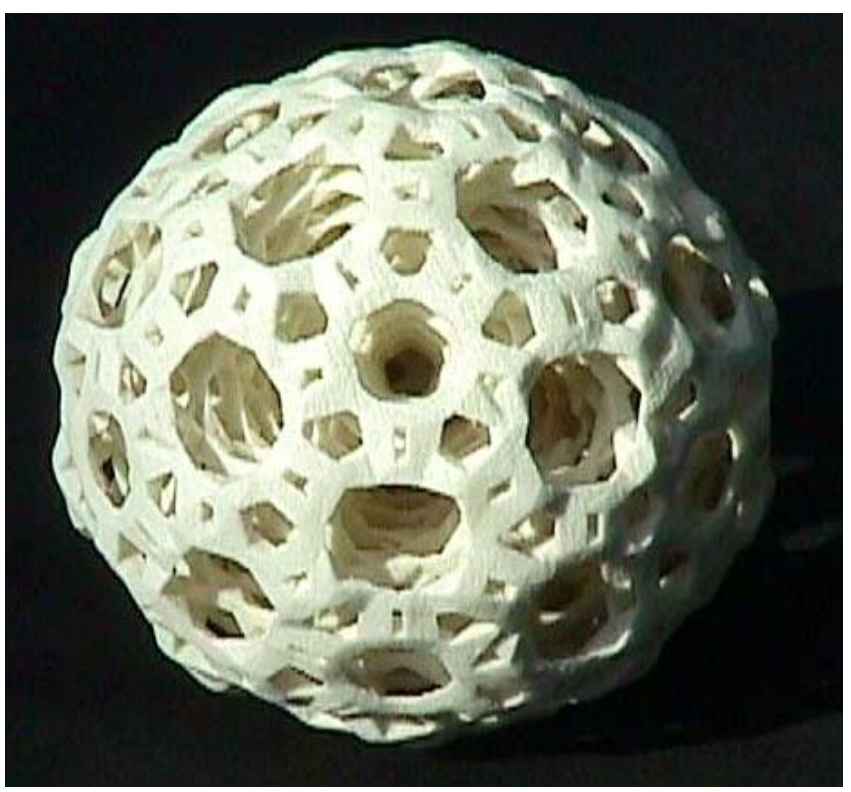

Figure 10. Deep Structure, 4 inches, 3D printing. [7,10] consisting of five nested concentric orbs. Each of the five has the same structure as the outer, most visible, orb: there are 30 large 12-sided oval openings, 12 smaller 10 -sided openings, 80 irregular hexagonal openings, and 120 small rectangular openings. Oval "corkscrew spirals" in the 12-sided openings connect the layers with each other. The concept is based on familiar concentric ivory spheres which are traditionally turned on a lathe and hand carved, with holes in each layer providing access to the inner layers.

Figure 11. Gonads of the Rich and Famous, two 3-inch balls, 3D printing. [7, 8, 13] Computer-generated models of novel polyhedra had their edges adapted into solid forms in different ways. In the first, the edges remain lace-like at the surface of the sphere, while in the second, they are carried to the center. In both, the thickness of each edge is proportional to its length, which helps to make them seem more organic. The name reflects, in part, the fact that these are currently very expensive to produce. As $3 \mathrm{D}$ printing costs come down in the future, ordinary people will enjoy such gonads. 